\title{
Macroadenoma de hipófisis descubierto incidentalmente. Indicaciones del tratamiento quirúrgico a propósito de dos casos
}

\author{
C. Maciá-Bobes; A. Ronzón-Fernández*; G. Castaño-Fernández y P. Botas-Cervero
}

Unidad de Endocrinología y *Unidad de Medicina de Familia. Hospital San Agustín. Avilés. Asturias.

\section{Resumen}

Los macroadenomas hipofisarios (de diámetro superior a $10 \mathrm{~mm}$ ) son poco frecuentes como hallazgos casuales, y su manejo diagnóstico y terapéutico no está bien definido. Los criterios más habituales para el tratamiento neuroquirúrgico son la afectación del campo visual, la hipersecreción de hormonas distintas a la prolactina, la constatación de crecimiento, o la apoplejía no silente. Presentamos dos casos en los que la indicación de cirugía se estableció en función de la edad -joven- de la paciente (caso número uno) y de la afectación del eje gonadal en un varón no subsidiario de tratamiento androgénico (caso número dos). Se discute el beneficio de incluir tales indicaciones quirúrgicas en el protocolo de evaluación de estas lesiones.

PALABRAS CLAVE: Incidentalomas hipofisarios. Macroadenomas hipofisarios. Tratamiento neuroquirúrgico.

Incidentally discovered pituitary macroadenoma. Neurosurgical treatment indications illutrated by two cases

\section{Summary}

Pituitary macroadenomas (more than $10 \mathrm{~mm}$ in diameter) are infrequent as casual findings and optimal management strategy for these tumours has not been established. Neurosurgical approach must be always considered in patients with visual field defects or with hormone-secreting adenomas (but prolactinoma), and in those with evidence of lesion's growth or if clinical pituitary apoplexy occurs. We present two cases in which surgical indication was based on patient's young age (case number one), and on hypogonadal status, in a male patient not suitable of androgen substitution (case number two). We also discuss the benefits of including such unusual indications for neurosurgical treatment into the incidentally discovered pituitary

Recibido: 04-01-05. Aceptado: 10-04-05. macroadenomas evaluation strategy.

KEY WORDS: Pituitary incidentalomas. Pituitary macroadenomas. Neurosurgical treatment

Introducción

Los procesos expansivos hipofisarios descubiertos casualmente en un estudio de imagen, indicado ante una situación clínica en la que no existe sospecha de enfermedad de la glándula, suelen recibir el nombre genérico de "incidentalomas hipofisarios". La prevalencia de estas lesiones como hallazgo mediante tomografía computarizada (TAC) o resonancia nuclear magnética (RNM) se aproxima al $10 \%{ }^{2}$, y en series autópsicas alcanza el $18 \%{ }^{22}$. Se trata, en la gran mayoría de los casos, de microadenomas de hipófisis (por definición, aquéllos con diámetro igual o inferior a $10 \mathrm{~mm}$ ), pero también es posible encontrar excepcionalmente tumores de otra estirpe (metastásicos, de células germinales, gliomas, craneofaringiomas), enfermedades inflamatorias (sarcoidosis, tuberculosis, histiocitosis X) y lesiones vasculares (aneurismas) ${ }^{11}$. Los macroadenomas hipofisarios (de diámetro superior a $10 \mathrm{~mm}$ ) son asimismo infrecuentes, con una prevalencia del $0,20 \%$ en población viva no seleccionada ${ }^{18}$, y aún menor en series autópsicas. La estrategia de manejo de estos "incidentalomas", tipo macroadenoma, es motivo de controversia en varios aspectos, entre otros los criterios de tratamiento quirúrgico.

Presentamos dos casos de macroadenomas hipofisarios descubiertos incidentalmente en los que se indicó cirugía, en función de la edad de la paciente, (caso número 1) y de la afectación del eje gonadal de un varón, no subsidiario de tratamiento androgénico (caso número 2). Ambas circunstancias, que se presentan con cierta frecuencia en la práctica clínica, constituyen indicaciones quirúrgicas atípicas (no contempladas por las recomendaciones de los expertos).

\section{Casos clínicos}

Caso número 1: Mujer de 32 años, con ciclos menstrua- 


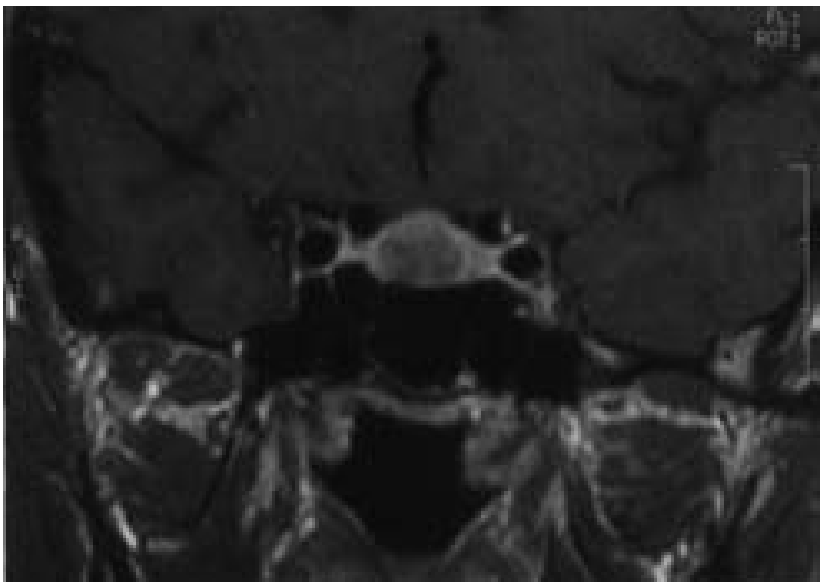

Figura 1. Imagen de resonancia nuclear magnética (T1, con contraste) en que se observa un adenoma hipofisario de $13 \times 10 \mathrm{~mm}$, intraselar, ligeramente lateralizado hacia la derecha.

les regulares, asintomática y sin tratamientos crónicos, a la que se le practicó una RNM craneal en febrero de 2002 tras un traumatismo craneoencefálico. Se objetivó un adenoma hipofisario de $13 \times 10 \mathrm{~mm}$, intraselar, lateralizado hacia la derecha. El campo visual y la exploración oftalmológica eran normales, y las determinaciones de hormonas hipofisarias también. En julio de 2002 el estudio de imagen era similar, así como el de enero de 2003 (figura 1), tras tratamiento con cabergolina $(0,5 \mathrm{mg}$ dos veces por semana) durante 3 meses. Ante la necesidad de un seguimiento indefinido, previsiblemente largo por la edad de la paciente, se acordó con ella la exéresis quirúrgica del macroadenoma no funcionante, que se efectuó de forma total y selectiva, por vía transesfenoidal sublabial, en noviembre de 2003. No se observó ningún déficit hormonal postoperatorio y la RNM de mayo de 2004 sólo mostraba cambios postquirúrgicos en el área de la silla turca, con hipófisis normal.

Caso número 2: Varón de 66 años, con depresión no tratada farmacológicamente, y con hiperplasia benigna de próstata que precisó biopsia en 3 ocasiones por cifras elevadas de antígeno prostático (PSA), al que se le descubrió un adenoma hipofisario de 19 x $18 \mathrm{~mm}$ en septiembre de 2003 en una TAC de conductos auditivos solicitada por episodios vertiginosos. Este hallazgo se confirmó mediante RNM en octubre de 2003 (figura 2). El paciente refería astenia intensa, sofocos, pérdida de fuerza muscular y disfunción eréctil completa en el último año. La campimetría era normal. El estudio hormonal hipofisario mostró una testosterona total de $0,19 \mathrm{ng} / \mathrm{mL}$ (valor normal (VN).: 2,98,0 ), una testosterona libre de $1,2 \mathrm{pg} / \mathrm{mL}$ (VN: 9-27), LH: 1,5 U/L (VN: 7-24), prolactina: $35 \mathrm{ng} / \mathrm{mL}$ (VN: 4,0-18,5), subunidad alfa libre de las hormonas glucoproteicas y resto de determinaciones normales. Se instauró tratamiento con

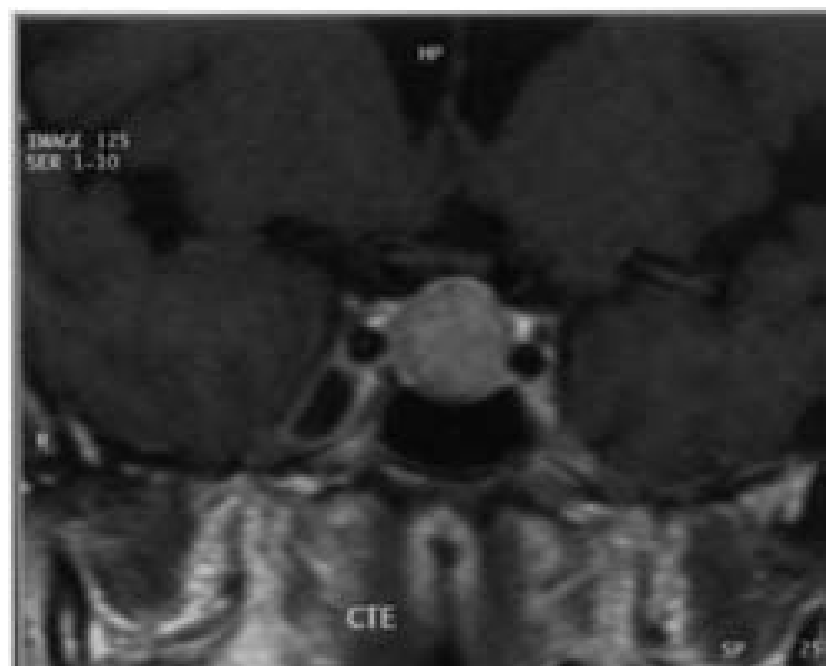

Figura 2. Imagen de resonancia nuclear magnética (T1, con contraste) en que se aprecia un adenoma hipofisario de $19 \times 18 \mathrm{~mm}$, que no llega a establecer contacto con el quiasma óptico.

bromocriptina ( $10 \mathrm{mg}$ al día) y 6 meses después el tumor hipofisario no se había reducido, la prolactina era de $0,5 \mathrm{ng} /$ $\mathrm{mL}$, la testosterona total de $0,4 \mathrm{ng} / \mathrm{mL}$, y la $\mathrm{LH}$ de $1,9 \mathrm{U} / \mathrm{L}$. La discreta hiperprolactinemia inicial se interpretó como secundaria a distorsión del tallo hipofisario. Se estableció el diagnóstico firme de hipogonadismo hipogonadotropo y se recomendó al paciente cirugía del macroadenoma no funcionante. En junio de 2004 se procedió a su resección por vía transesfenoidal transoral, y 3 meses después, sin tratamiento hormonal, la testosterona total era de 2,0, la LH de 4,0, la prolactina de 8,2 , y el resto del estudio hipofisario era normal. En noviembre de este año, en la RNM, no se veían restos macroscópicos del adenoma. El paciente se encontraba subjetivamente mucho mejor, sin astenia, sin sofocos, con erecciones satisfactorias y menos deprimido, aun cuando la producción de andrógenos no se había normalizado por completo.

\section{Discusión}

Los macroadenomas de hipófisis descubiertos incidentalmente son poco frecuentes ${ }^{18}$, aunque hay datos discordantes; por ejemplo, un estudio de la Universidad de Dusseldorf de $1999^{8}$ encuentra un $37,3 \%$ de macroadenomas en una serie de 67 "incidentalomas". No existe una sistematización de los procederes diagnósticos y terapéuticos, ni del plan de seguimiento en caso de tratamiento conservador.

Por lo que se refiere a la secreción hormonal, la prueba diagnóstica más rentable es la determinación de prolactina, y el resto de las hormonas hipofisarias, según criterios de coste-efectividad, se investigaría sólo si los datos clínicos 
sugiriesen disfunción ${ }^{13}$. Sin embargo, en la práctica, el estudio funcional incluye una media de 11 pruebas en el Reino Unido y de 10 en Estados Unidos ${ }^{12}$, y parece adecuado excluir sistemáticamente déficits de ACTH, TSH $\mathrm{y}$ gonadotropinas, muy frecuentes ${ }^{8,23}$. Existe un acuerdo general sobre la necesidad de descartar alteraciones campimétricas ${ }^{10}$, puesto que tanto el compromiso visual como las manifestaciones neurológicas por efecto masa son indicaciones indiscutibles de cirugía ${ }^{19}$. Otra cuestión debatida es la periodicidad de las pruebas de imagen y la duración del seguimiento: 1-2-5 años según Torres et al. ${ }^{23}$; 0,5-1-1,5-2 años y luego de modo anual indefinido según Sanno et al. ${ }^{21}$; 1-2-3 años y luego a intervalos más amplios no precisados según Aron et al. ${ }^{2}$. El crecimiento del tumor, que ocurrió en un $15-20 \%$ de los casos, o la apoplejía, en un $0,4 \%$, en una serie de 248 pacientes con un seguimiento medio de 5 años ${ }^{21}$, constituyen en general criterios quirúrgicos mayores.

Así pues, el punto de vista más conservador reservaría la cirugía para los tumores con hipersecreción hormonal (excepto prolactinomas), o con afectación de la vía óptica, para los que experimenten un aumento de tamaño en el seguimiento o los que sufran una apoplejía clínicamente significativa $^{2,19}$. Es preciso tener en cuenta que cada vez se publican más casos de apoplejía "silente" ${ }^{20}$ o con buena evolución en régimen médico ${ }^{1}$; los fenómenos apopléticos, por otra parte, pueden conducir a una resolución de la hipersecreción hormonal ${ }^{15} \mathrm{o}$ a una desaparición del tumor ${ }^{25}$. La disminución de tamaño con tratamiento médico de los adenomas productores de $\mathrm{GH}^{4}$ o $\mathrm{TSH}^{3}$ es posible, por lo que los análogos de somatostatina podrían constituir una alternativa a la neurocirugía en casos de difícil resección (e improbable curación), o en pacientes ancianos o deteriorados $^{9}$. En cambio, los adenomas no funcionantes rara vez (10\%) experimentan reducción de masa con tratamiento médico ${ }^{5}$, por lo que la expectativa de éxito debe ser limitada. Los pacientes presentados tomaron bromocriptina $\mathrm{o}$ cabergolina, a dosis bajas o medias, durante 3 a 6 meses, sin resultados apreciables en la RNM de hipófisis; en realidad, el tratamiento se enfocó como un intento de agotar otras vías y como elemento de persuasión para la cirugía.

La actitud terapéutica más intervencionista propondría la exéresis neuroquirúrgica de todos los "incidentalomas" hipofisarios tipo macroadenoma. No goza de gran aceptación, ante la evidencia de que la historia natural de estos tumores es relativamente benigna ${ }^{7,19}$, y el crecimiento en períodos de tiempo intermedios ocurre en menos de una quinta parte de $\operatorname{los} \operatorname{casos}^{21}$. La hipofunción de la glándula, referida al menos a un eje hormonal (el gonadal es el de afectación más prevalente $)^{8}$, no constituye un criterio de indicación quirúrgica para la mayoría de los autores, que simplemente recomiendan tratamiento médico sustitutivo $^{2,7,17,21}$ (con alguna excepción ${ }^{23}$ ) a pesar de que la cirugía consigue la recuperación de al menos un eje hormonal en un $35 \%$ de los macroadenomas no funcionantes con déficits preoperatorios $^{24}$.

El paciente del caso número 2, afecto de un hipogonadismo hipogonadotropo, que repercutía sin duda en su calidad de vida (disfunción eréctil, astenia, debilidad muscular), además de aumentar el riesgo de osteoporosis, no era candidato a tratamiento androgénico, por contraindicación expresa del Servicio de Urología: la administración de testosterona, incluso en las modalidades más "fisiológicas" (parches o gel transdérmicos), condiciona un grado de hiperplasia prostática muy superior al de la testosterona endógena ${ }^{16}$, por lo que debe desaconsejarse en casos como el referido, con varias biopsias prostáticas en su haber. Parece razonable pues que el déficit hormonal hipofisario, especialmente en los casos en que el tratamiento médico sustitutivo sea imposible, se admita como situación tributaria de neurocirugía.

Tampoco la edad es considerada como criterio quirúrgico, a pesar de que un paciente joven (como la del caso número 1), en seguimiento, sería sometido a múltiples exploraciones radiológicas y campimétricas a lo largo de su vida, incluso en caso de no presentar intercurrencias tales como embarazos, cefaleas, pérdidas visuales de cualquier origen, necesidad de anticoagulación, etc. En el campo de la Endocrinología, además, la edad (inferior a 50 años) constituye por sí sola un criterio para la cirugía en el hiperparatiroidismo primario asintomático ${ }^{6}$.

La mortalidad de las intervenciones de hipófisis por vía transesfenoidal, en manos de un cirujano experto, ronda el $0,5 \%$ y la morbilidad mayor es inferior al $1,5 \%{ }^{14}$. Puesto que los datos proceden de series amplias, que incluyen reintervenciones, adenomas funcionantes y casos históricos, probablemente el riesgo para un paciente "actual" con un macro-incidentaloma no funcionante sea considerablemente menor, e inferior, por ejemplo, al riesgo de sufrir una apoplejía hipofisaria si la expectativa de vida es larga.

Parece pues que el cociente riesgo/beneficio sería favorable a la intervención en pacientes jóvenes al suprimir la necesidad de seguimiento prolongado. Muy probablemente, la cirugía resulte también rentable desde el punto de vista coste-efectividad, aunque no se dispone de estudios que avalen este extremo.

\section{Conclusión}

Aunque, habitualmente, sólo se consideran indicaciones de tratamiento quirúrgico de los "incidentalomas" hipofisarios el tamaño o el crecimiento, la hipersecreción hormonal (distinta a la prolactina) o la apoplejía con significación clínica, nuestra opinión es que debería discutirse la conveniencia de incluir como criterios para la cirugía tanto la edad (joven) de los pacientes como los déficits hor- 
monales, especialmente en los casos en que el tratamiento sustitutivo resulte difícil o imposible.

\section{Bibliografía}

1. Aguirre Sánchez-Covisa, M., Hernández González, A., Chamorro Prado, R., Sanabria Pérez, M.C.: Apoplejía hipofisaria: 3 casos con buena evolución sin tratamiento quirúrgico. Endocrinol Nutr 1998; 45: 64-67.

2. Aron, D.C., Howlett, T.A.: Pituitary incidentalomas. Endocrinol Metab Clin North Am 2000; 29: 205-221.

3. Beck-Peccoz, P., Persani, L.: Medical management of thyrotropin-secreting pituitary adenomas. Pituitary 2002; 5: 83-88.

4. Ben-Shlomo, A., Melmed, S.: Acromegaly. Endocrinol Metab Clin North Am 2001; 30: 565-583.

5. Bevan, J.S., Webster, J., Burke, C.W., Scanlon, M.F.: Dopamine agonists and pituitary tumor shrinkage. Endocr Rev 1992; 13: 220-240.

6. Bilezikian, J.P., Silverberg, S.J.: Asymptomatic primary hyperparathyroidism. N Engl J Med 2004; 350: 1746-1751.

7. Donovan, L.E., Corenblum, B.: The natural history of the pituitary incidentaloma. Arch Intern Med 1995; 155: 181183.

8. Feldkamp, J., Santen, R., Harms, E., Aulich, A., Modder, U., Scherbaum, W.A.: Incidentally discovered pituitary lesions: high frequency of macroadenomas and hormone-secreting adenomas - results of a prospective study. Clin Endocrinol (Oxf) 1999; 51: 109-113.

9. Fraioli, B., Pastore, F.S., Signoretti, S., De Caro, G.M., Giuffre, R.: The surgical treatment of pituitary adenomas in the eight decade. Surg Neurol 1999; 51: 261-266.

10. Fujimoto, N., Saeki, N., Miyauchi, O., Adachi-Usami, E.: Criteria for early detection of temporal hemianopsia in asymptomatic pituitary tumor. Eye 2002; 16: 731-738.

11. Gsponer, J., De Tribolet, N., Deruaz, J.P., et al.: Diagnosis, treatment, and outcome of pituitary tumors and other abnormal intrasellar masses. Retrospective analysis of 353 patients. Medicine (Baltimore) 1999; 78: 236-269.

12. Howlett, T.A., Como, J., Aron, D.C.: Management of pituitary incidentalomas. A survey of British and American endocrinologists. Endocrinol Metab Clin North Am 2000; 29: 223-230.

13. King, J.T., Justice, A., Aron, D.C.: Management of incidental pituitary macroadenomas: a cost-effectiveness analysis. J Clin Endocrinol Metab 1997; 82: 3625-3632.

14. Laws, E.R.Jr., Thapar, K.: Pituitary surgery. Endocri- nol Metab Clin North Am 1999; 28: 119-131.

15. Marazuela, M., Lucas, Y., Estrada, J., Barceló, B.: Curación espontánea de una acromegalia después de una apoplejía hipofisaria; presentación de dos casos. Med Clin (Barc) 1993; 100: 556-557.

16. Martínez Portillo, F.J., Cueva Martínez, A., Martín Braun, P., Fernández Arancibia, M.I., Junemann, K.P., Alken, P.: Sustitución con testosterona en pacientes con hipogonadismo. Arch Esp Urol 2002; 55: 827-838.

17. Molitch, M.E.: Pituitary incidentalomas. Endocrinol Metab Clin North Am 1997; 26: 725-740.

18. Nammour, G.M., Ybarra, J., Naheedy, M.H., Romeo, J.H., Aron, D.C.: Incidental pituitary macroadenoma: a population-based study. Am J Med Sci 1997; 314: 287-291.

19. Nishizawa, S., Ohta, S., Yokoyama, T., Uemura, K.: Therapeutic strategy for incidentally found pituitary tumors ("pituitary incidentalomas"). Neurosurgery 1998; 46: 13441350.

20. Onesti, S.T., Wisnieewski, T., Post, K.D.: Clinical versus subclinical pituitary apoplexy: presentation, surgical management, and outcome in 21 patients. Neurosurgery 1990; 26: 980-986.

21. Sanno, N., Oyama, K., Tahara, S., Teramoto, A., Kato, Y.: A survey of pituitary incidentaloma in Japan. Eur J Endocrinol 2003; 149: 123-127.

22. Teramoto, A., Hirakawa, K., Sanno, N., Osamura, Y.: Incidental pituitary lesions in 1000 unelected autopsy specimens. Neuroradiology 1994; 193: 161-164.

23. Torres, Y., Acebes, J.J., Soler, J.: Incidentaloma hipofisario: evaluación y abordaje terapéutico en la actualidad. Endocrinol Nutr 2003; 50: 153-155.

24. Webb, S.M., Rigla, M., Wagner, A., Oliver, B., Bartumeus, F.: Recovery of hypopituitarism after neurosurgical treatment of pituitary adenomas. J Clin Endocrinol Metab 1999; 84: 3696-3700.

25. Weiss, R.E.: Empty sella following spontaneous resolution of a pituitary macroadenoma. Horro Res 2003; 60: 4952.

Maciá-Bobes, C.; Ronzón-Fernández, A.; Castaño-Fernández, G.; Botas-Cervero, P.: Macroadenoma de hipófisis descubierto incidentalmente. Indicaciones del tratamiento quirúrgico a propósito de dos casos. Neurocirugía 2006; 17: 538-541.

Correspondencia postal: Carmen Maciá Bobes. C/ Pablo Laloux 13, $6^{\circ}$ A-Sur. 33405 Salinas. Castrillón. Asturias 